\title{
Low Levels of Estrogen Significantly Diminish Axonal Sprouting after Entorhinal Cortex Lesions in the Mouse
}

\author{
Inga Kadish ${ }^{1}$ and Thomas van Groen ${ }^{1,2}$ \\ ${ }^{1}$ Department of Neuroscience and Neurology, University of Kuopio, and ${ }^{2}$ Department of Neurology, \\ Kuopio University Hospital, FIN 70211 Kuopio, Finland
}

\begin{abstract}
This study tested the hypothesis that estrogen enhances axonal sprouting in the hippocampal formation in the female mouse. The entorhinal cortex was unilaterally lesioned with ibotenic acid in control mice and in ovariectomized mice that were treated with a high dose of, a moderate dose of, or zero estrogen supplementation pellets. Four weeks later the density of staining for synaptophysin immunoreactivity and acetylcholinesterase (AChE) histochemistry was measured in the molecular layer of the dentate gyrus. In control mice, lesions of the lateral part of the entorhinal cortex increased synaptophysin and acetylcholinesterase staining (i.e., indicative of axonal sprouting) in the outer one-third of the molecular layer of the
\end{abstract}

After brain injury, several growth processes take place. For instance, the neurons with axons that have been severed respond with growth, or, in the area that is denervated, collateral sprouting, i.e., terminal proliferation (the formation of new terminals), and reactive synaptogenesis take place (Steward, 1991; Deller and Frotscher, 1997). A number of growth processes underlie this, including growth of axons into the area that is denervated and collateral sprouting by local surviving axons (i.e., terminal proliferation) (Deller and Frotscher, 1997). Many studies have demonstrated that, after entorhinal cortex ablations, the dentate gyrus of the hippocampus shows an early phase of degeneration of the lesioned axons and terminals, followed by a sprouting response of surviving axons, (Cotman and Nadler, 1978; Steward, 1991), and thus this system has often been used as a model to elucidate the mechanisms underlying neuronal plasticity after brain injury. This model is especially useful, because the entorhinal cortex axons terminate in precise and organized bands in the outer one-third (lateral entorhinal) and middle one-third (medial entorhinal) of the molecular layer of the dentate gyrus. Thus most alterations in these layers after an entorhinal cortex lesion can be attributed to the ingrowth or sprouting of homotypic collateral axons (Frotscher et al., 1997).

Studies have suggested that during mammalian development, estradiol exerts trophic effects (for review, see Beyer, 1999). In recent years it has been shown that the adult brain also remains plastic and that sex hormones continue to regulate its structure and plasticity (García-Segura et al., 1994; Dubal et al., 1999). Experimental studies on animal models have shown that the

\footnotetext{
Received Dec. 18, 2001; revised Feb. 12, 2002; accepted March 1, 2002.

This study was supported by TEKES project 40043/01, and the Academy of Finland.

Correspondence should be addressed to Thomas van Groen, Department of Neuroscience and Neurology, Canthia Building, Harjulantie 1D, University of Kuopio, FIN 70211 Kuopio, Finland. E-mail: thomas.vangroen@uku.fi. Copyright (C) 2002 Society for Neuroscience $0270-6474 / 02 / 224095-08 \$ 15.00 / 0$
}

dentate gyrus. Mice receiving high and moderate estrogen supplementation displayed the same sprouting response; however, in ovariectomized mice the sprouting response was significantly reduced (to nearly nothing). Thus, in ovariectomized compared with control mice the lesion-induced sprouting response is severely blunted, and this effect is reversed by estrogen supplementation. Together, these findings suggest that estrogen plays a prominent role in promoting neuronal plasticity and remodeling in the dentate gyrus.

Key words: estrogen; mice; hippocampal formation; limbic system; sex hormone; female

gonadal steroids, estradiol (Garcia-Estrada et al., 1993), testosterone, and progesterone (García-Segura et al., 1994), modulate the response of nervous tissue to injury. Estradiol has been shown to participate in reorganization of denervated nervous tissue (McEwen and Alves, 1999; García-Segura et al., 2001), and estrogen has been shown to modulate the sprouting of axons after brain lesion in the rat (Loy and Milner, 1980; Morse et al., 1986, 1992). Thus far, only one study (Stone et al., 1998) has yet assessed the role of estrogen in neuronal plasticity in the mature female mouse cortex; however, this is of import for two reasons. First, the distribution of estrogen receptors differs between rats and mice (Shugrue et al., 1997), and second, we (and many others) are studying transgenic mice in our current studies (Kadish et al., 2002).

Therefore, the present study examined the effects of estrogen depletion on axonal sprouting in the mouse dentate gyrus. The entorhinal cortex was unilaterally lesioned with ibotenic acid in control and ovariectomized mice (with or without estrogen supplementation), and the density of synaptophysin and acetylcholinesterase (AChE) staining was measured in the molecular layer of the dentate gyrus.

\section{MATERIALS AND METHODS}

Animals. Adult, female mice (C57BL/6J strain; National Laboratory Animal Center, Kuopio, Finland) were housed two to three per cage at constant humidity $(60 \pm 5 \%)$, temperature $\left(22 \pm 1^{\circ} \mathrm{C}\right)$, and light cycle $(6$ A.M.-6 P.M.). After surgery all animals (including controls) were housed individually. At 3 months of age, 10 mice were ovariectomized, 10 mice were ovariectomized and received estrogen supplementation with a moderate dose of estrogen, 10 mice were ovariectomized and received a high dose of estrogen, and 15 mice served as surgical controls. After recovery, i.e., 1 week later, all animals received unilateral entorhinal cortex lesions using ibotenic acid, except for five sham injection control animals. All procedures were approved by the Animal Use and Care Committee of the University of Kuopio, the State Provincial Office of Eastern Finland, and followed the guidelines of the European Communities Council Directive of 24 November 1986 (86/609/EEC). 
Ovariectomy. For ovariectomy (OE), the mice were anesthetized with a mixture of a sodium pentobarbital and chloral hydrate solution $(50 / 50$; $36 \mathrm{mg} / \mathrm{kg}$, i.p.). The animal was placed on its abdomen, and the skin on the back was shaved and sterilized. A small dorsal midline incision was made in the skin, approximately in the middle (anteroposterior) of the back, and then a muscle incision was made approximately halfway down the side of the body. The ovaries were visualized in the abdominal cavity and retracted from the body using a forceps (they were removed by holding onto the periovarian fat). The junction between the fallopian tube and the uterine horn was cut, the ovary was removed, the uterine horn was replaced in the abdominal cavity, and the wound was closed. After surgery (i.e., on the same day), a pellet containing $17 \beta$-estradiol (Innovative Research of America, Sarasota, FL) was placed under the skin at the back of the neck. These pellets released a steady flow of the hormone for the duration of the experiment (i.e., $60 \mathrm{~d}$ release pellets). The moderate $(0.18 \mathrm{mg}$ per pellet) dose pellets resulted in a moderate level of $75-100 \mathrm{pg} / \mathrm{ml}$ of estrogen in the blood; the high $(0.72 \mathrm{mg}$ per pellet) dose pellets resulted in a high level of $300-400 \mathrm{pg} / \mathrm{ml}$ of estrogen (Innovative Research of America) in the blood. To control for the efficacy of the ovariectomy and estrogen supplementation after the animals were killed, the weight of the uterus was measured.

Entorhinal cortex lesions. The mice were reanesthetized with a mixture of a sodium pentobarbital and chloral hydrate solution $(50 / 50 ; 36 \mathrm{mg} / \mathrm{kg}$, i.p.) and placed in a stereotaxic frame, and the needle $(90 \mu \mathrm{m}$ tip diameter) of a Hamilton $0.5 \mu \mathrm{l}$ microsyringe was stereotaxically lowered to the entorhinal cortex. In 40 animals, a solution of ibotenic acid (10 $\mathrm{mg} / \mathrm{ml})$ in PBS, $\mathrm{pH} 7.4$, was unilaterally injected $(2 \times 150 \mathrm{nl}$; i.e., two injections on one side of the brain) into the entorhinal cortex at a rate of $50 \mathrm{nl} / \mathrm{min}$. After the injection, the syringe was left in place for another 5 min before retraction. In five mice, the same procedure was followed, but only vehicle was injected. Four weeks after the lesions, the animals were reanesthetized and transcardially perfused with $50 \mathrm{ml}$ of buffered saline,

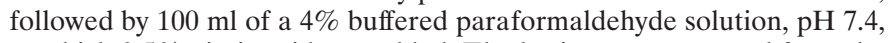
to which $0.5 \%$ picric acid was added. The brains were removed from the skull and stored in the fixative for $4 \mathrm{hr}$, and thereafter they were transferred to a $30 \%$ sucrose solution for cryoprotection. Three series of ( 1 in 6$)$ coronal sections $(35 \mu \mathrm{m})$ were cut on a freezing microtome. The first series of sections was mounted on gelatin-coated slides immediately, stained with cresyl violet, and coverslipped. The second series was histochemically stained for AChE, mounted, and coverslipped (van Groen and Wyss, 1992). The third series was immunohistochemically stained for synaptophysin. The sections were rinsed overnight in a solution of Tris-buffered saline (TBS), and then the series of sections was transferred to a solution containing the primary antibody (mouse antisynaptophysin; 1:1000; Sigma, St. Louis, MO); this solution consisted of TBS with $0.5 \%$ Triton X-100 added (TBS-T). After incubation in this solution for $18 \mathrm{hr}$ on a shaker table at room temperature $\left(20^{\circ} \mathrm{C}\right)$ in the dark, the sections were rinsed three times in TBS-T and transferred to the solution containing the secondary antibody (goat anti-mouse-biotin; Sigma). After $2 \mathrm{hr}$, the sections were rinsed three times with TBS-T and transferred to the tertiary antibody (part of the mouse ExtrAvidin staining kit; Sigma); after staining with the kit and rinsing, the sections were incubated for $\sim 3$ min with Ni-enhanced diaminobenzidine (DAB) [10 mg DAB in $20 \mathrm{ml} 0.1 \mathrm{M}$ phosphate buffer, $30 \mu \mathrm{H}_{2} \mathrm{O}_{2}(30 \%)$, pH 7.4, with $1 \mathrm{ml}$ of a $15 \%$ ammonium Ni-sulfate solution added]. The stained sections were mounted on slides and coverslipped. The stained material was inspected under light microscopy. To control for the specificity of immunocytochemical staining, the primary antibody was omitted in a few animals.

Measurements. The appropriate sections and areas of the dorsal hippocampus were digitized using a Nikon Coolpix 990 camera, and the images were converted to gray scale using the Paint Shop Pro 7 program. To avoid changes in lighting that might affect measurements, all images were acquired in one session. The optical density was measured in the appropriate band of the molecular layer (i.e., the outer molecular layer) (see Fig. $1 E, F$ ) of the ipsilateral (to the lesion) and contralateral dentate gyrus and in ipsilateral and contralateral area CA1 (in the same sections) of the hippocampus using the ScionImage (NIH) program. These measurements were done on the same section or on the adjacent, simultaneously stained section. All density measurements were done in triplicate, i.e., by measuring a standardized area at three lateromedial positions at three different levels in the dorsal hippocampus (see Fig. $1 C, E)$, but at similar lateromedial positions in the dentate gyrus and in area CA1 (see Fig. $1 E$ ). Density measurements were performed by an investigator who was blind to the treatment of the animals. For analysis the optical density measurements were converted to relative densities; i.e., the density of synaptophysin and AChE staining of the molecular layer of the contralateral dentate gyrus was set as $100 \%$. Furthermore, the width of the molecular layer (and inner molecular layer) of the dentate gyrus and of stratum lacunosum-moleculare and stratum radiatum of area CA1 was measured (see Fig. $1 F$ ). Again, all measurements were done in triplicate, i.e., measuring the width at three levels of the dorsal hippocampus but at the same lateromedial position in the dentate gyrus and in area CA1 (see Fig. $1 C, F$ ). This was necessary because the width of these layers varies with the lateromedial position of the measurement in the hippocampus (see Fig. $1 E$ ). Data were analyzed by Student's paired $t$ test (ipsilateral versus contralateral) and by ANOVA (SPSS version 10.0; between groups), and post hoc tests (Tukey and Scheffe) were performed to determine the source of a significant main effect or interaction.

\section{RESULTS}

\section{Animals}

All animals that received $\mathrm{OE}$ had uterine weights that were significantly lower than the control mice $(18 \pm 2$ and $76 \pm 2 \mathrm{mg}$, respectively; $p<0.01)$. The estradiol-treated animals had uterine weights that were significantly higher than the control mice [moderate dose estradiol supplementation (M-ES), $p<0.05$; high dose estradiol supplementation (H-ES), $p<0.01]$. The animals that received a moderate dose of estradiol had uterine weights of $125 \pm 5 \mathrm{mg}$ that were lower than those of the animals that received the high dose of estradiol (uterine weights of $148 \pm 6$ $\mathrm{mg} ; p<0.05)$.

Accordingly, the entorhinal cortex lesioned animals were divided into four groups: entorhinal lesion control (EControl; $n=$ 10); OE $(n=8)$; OE + M-ES $(n=8)$; and OE + H-ES $(n=8)$. Because there were no differences in either the synaptophysin or AChE staining density between ipsilateral and contralateral areas in the hippocampus in the five sham lesion animals, these animals were not included in the analysis.

\section{Entorhinal cortex lesion}

The ibotenic acid injection protocol used in this study reliably resulted in partial lesions of the entorhinal cortex. In nearly all cases, the caudal, lateral part of the lateral entorhinal area was lesioned; in five animals, a small part of the lateral part of the medial entorhinal area was also lesioned (Fig. $1 A, B$ ). There were no significant differences in the size of the lesions between the groups. However, in two animals the entorhinal cortex lesion was very small, and in another two animals the lesion encroached significantly on the hippocampal formation; the data from these four animals were removed from the study. We chose to use partial lesions of the entorhinal cortex because the projection of the entorhinal cortex to the contralateral dentate gyrus is practically nonexistent in the mouse (van Groen et al., 2002) and would therefore not sprout. Furthermore, in these experiments we wanted to mimic the partial denervation with its concomitant homotypic sprouting of the hippocampus that is present in early Alzheimer's disease (Hyman et al., 1986, 1988; Kadish et al., 2002).

In normal mice, 4 weeks after the lateral entorhinal area lesions there was an increase in the density of staining for synaptophysin and $\mathrm{AChE}$ in the outer one-third of the molecular layer of the dentate gyrus ipsilateral to the lesion (Figs. 1, 2). The lesions of the lateral entorhinal area resulted in changes in the density of labeling in the outer one-third of the molecular layer of the dentate gyrus (i.e., lateral perforant path endings), where the axons from the lateral entorhinal area terminate (Fig. 2). In contrast, the lesions that included part of the medial entorhinal 
A
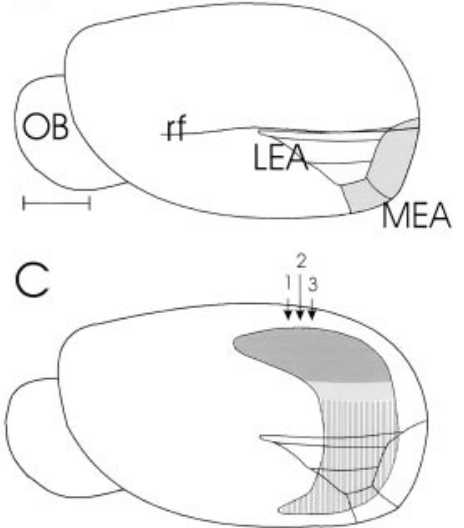

E

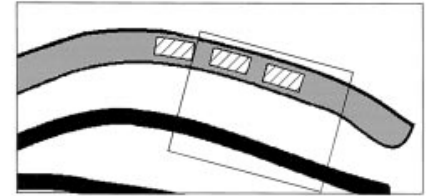

B

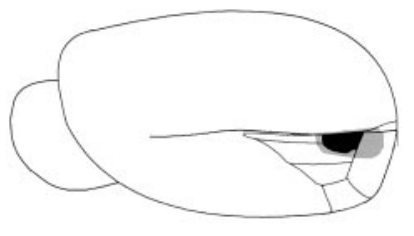

$\mathrm{D}$

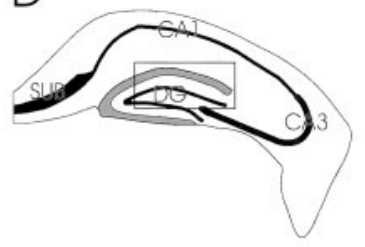

F

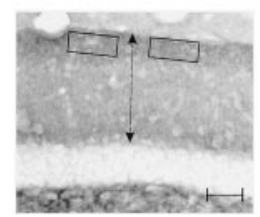

Figure 1. Three schematic drawings of the mouse brain. $A$, Cortex of the mouse indicating the position of the entorhinal cortex: dark shading, medial entorhinal area $(M E A)$; light shading, lateral entorhinal area $(L E A) . B$, Cortex of the mouse indicating the size and position of the lesions in the entorhinal cortex: dark shading, smallest lesion; light shading, largest lesion. $C$, Cortex of the mouse indicating the position of the hippocampus, with the shaded areas demonstrating the area involved in degeneration and regeneration; the striped area indicates the noninvolved part of the hippocampus. The three arrows indicate the position of the three sections through the hippocampus that were used for measuring the density. $D$, Coronal section through the hippocampus indicated by arrow 2; the shaded band in the outer molecular layer indicates the area of change after the lesion. E, Boxed area of $D$ at higher magnification; the striped boxes indicate the position of the three areas that were used for measurement of density changes. $F$, Boxed area in $E$ stained for synaptophysin. The two-headed arrow indicates the place of the length measurement. $O B$, Olfactory bulb; $r f$, rhinal fissure; $D G$, dentate gyrus; $C A 1$, cornu ammonis 1 ; $C A 3$, cornu ammonis $3 ; S U B$, subiculum. Scale bar (shown in $A$ for $A-C$ ): $1 \mathrm{~mm} ; F, 50 \mu \mathrm{m}$.

area also resulted in changes in the density of labeling in the middle one-third of the molecular layer of the dentate gyrus.

\section{Measurements}

The data of the measurements of the synaptophysin staining density demonstrated that the change in synaptophysin staining density was confined to the ipsilateral dentate gyrus. No measurable changes in density of staining were present in ipsilateral stratum lacunosum-moleculare of area CA1 (where the entorhinal axons also terminate) or in contralateral CA1, or in any other areas of the ipsilateral or contralateral hippocampus (Figs. 2, 3). Furthermore, it should be noted that the increase in staining density for synaptophysin was also reflected in material stained for AchE; i.e., this material also showed an increase in density of staining (Figs. 2, 4). Again, no changes in density of AChE staining were present in any other area of the ipsilateral or contralateral hippocampus (Fig. 2).

To avoid problems in the interpretation of our data, because shrinkage of the molecular layer would affect the synaptophysin staining density measurements, we measured the width of the dentate gyrus molecular layer (and the width of stratum lacunosum-moleculare/stratum radiatum of area CA1). None of
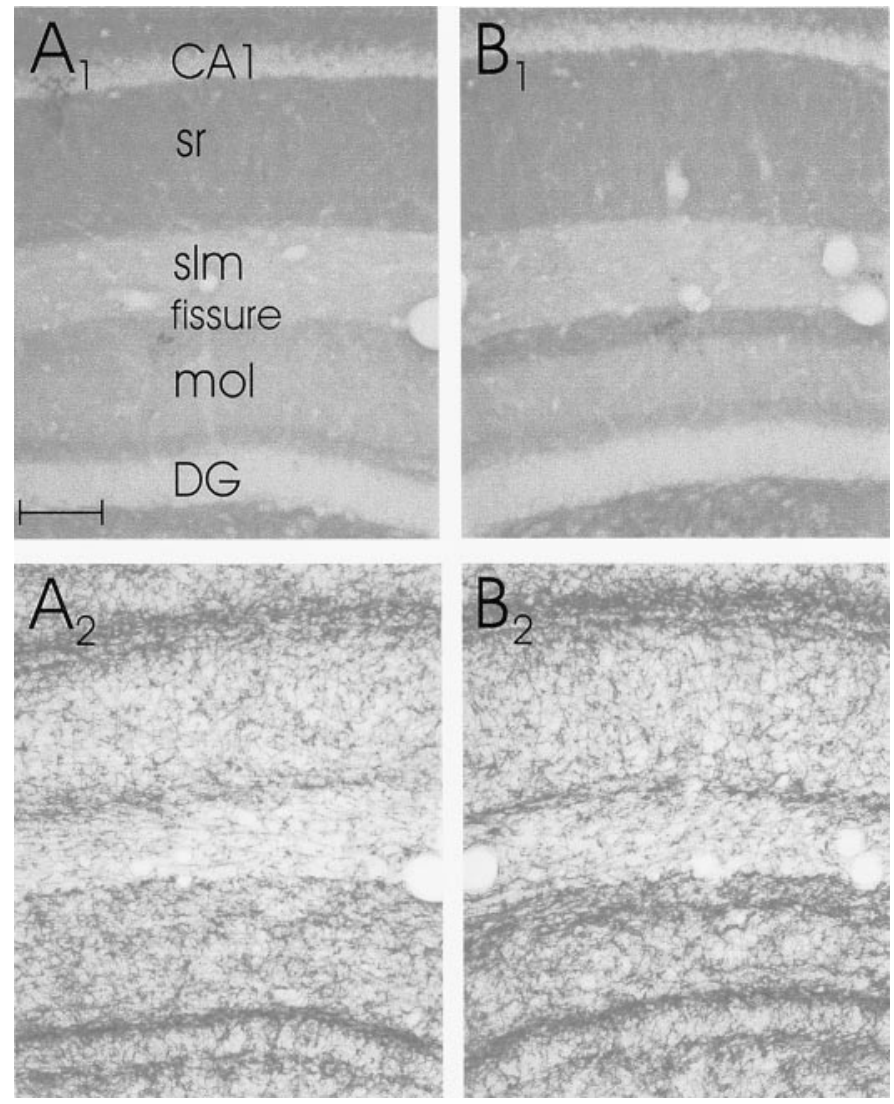

Figure 2. Four high-power photomicrographs of the dorsal hippocampus after an ipsilateral entorhinal cortex lesion in an EControl animal. $A$, Contralateral to the lesion; $B$, ipsilateral to the lesion. $A_{1}, B_{1}$, Synaptophysin-tained sections; $A_{2}, B_{2}$, adjacent sections stained for AChE. $D G$, Dentate gyrus; $s r$, stratum radiatum; $s l m$, stratum lacunosum moleculare; $\mathrm{mol}$, molecular layer. Scale bar (shown in $A_{1}$ for all photomicrographs): $100 \mu \mathrm{m}$.

the groups of mice showed a change in the width of the dentate gyrus molecular layer or in the width of stratum lacunosummoleculare + stratum radiatum of area CA1 (Table 1 ).

The data demonstrate that in EControl mice, 4 weeks after the lateral entorhinal area lesions there was a significant increase (from $100 \%$ to $119.5 \pm 4.4 \%$; $p<0.001$ ) (Fig. 5) in the density of staining for synaptophysin (i.e., sprouting) in the outer one-third of the dentate gyrus molecular layer ipsilateral to the lesion (Figs. $3,5)$. Both groups of mice that received ES displayed a similar sprouting response after the entorhinal cortex lesion [M-ES from $100 \%$ to $121.3 \pm 3.8 \%(p<0.01)$ and H-ES from $100 \%$ to $126.0 \pm 3.8 \%(p<0.01)$, respectively] (Fig. 5). However, in mice that were ovariectomized and did not receive estrogen supplementation (i.e., the OE group), the sprouting response was reduced substantially [i.e., no change, $100 \%$ and 100.1 substantially $\pm 0.5 \%$ (Figs. 3, 5) compared with all other groups; $\left.F_{(3,30)}=6.75 ; p<0.001\right]$. No significant changes in density of synaptophysin staining were present in stratum lacunosum-moleculare of ipsilateral area CA1 (where entorhinal axons also terminate) compared with contralateral CA1 (Figs. 3, 5).

The AChE staining data demonstrate that in EControl mice, 4 weeks after the lateral entorhinal area lesions there was an increase (from $100 \%$ to $126.7 \pm 5.9 \% ; p<0.05$ ) (Fig. 5) in the density of staining for $\mathrm{AChE}$ in the outer one-third of the dentate 

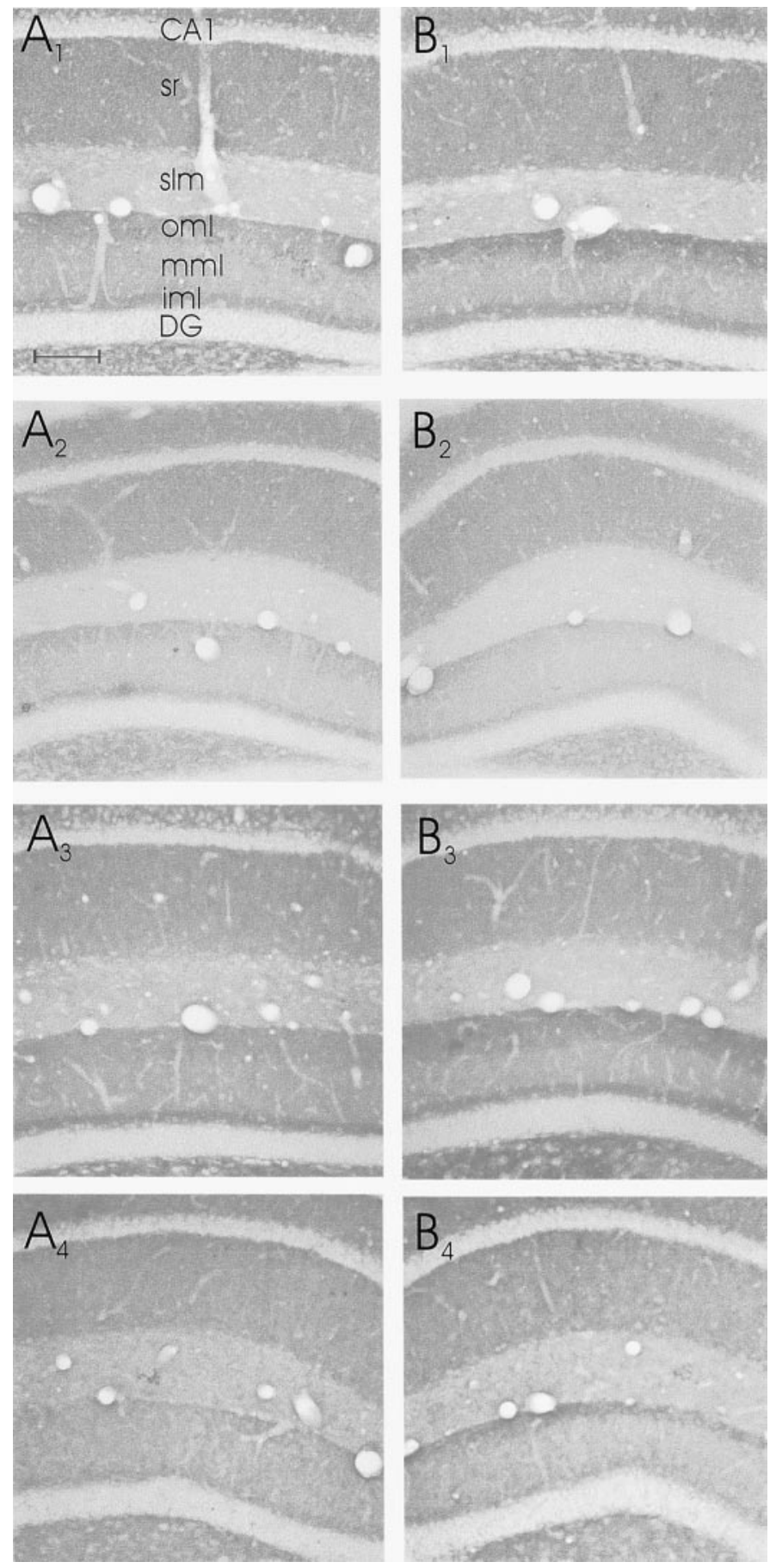

Figure 3. Eight high-power photomicrographs of synaptophysin-stained sections through the dorsal hippocampus. $A$, Contralateral to the entorhinal cortex lesion; $B$, ipsilateral to the entorhinal cortex lesion. $A_{1}, B_{1}$, Sections of a EControl mouse; $A_{2}, B_{2}$, sections from an OE mouse. $A_{3}, B_{3}$, Sections stained for synaptophysin from an M-ES mouse; $A_{4}, B_{4}$, sections from an H-ES mouse. $D G$, Dentate gyrus; iml, inner molecular layer; $\mathrm{mml}$, middle molecular layer; oml, outer molecular layer. Scale bar (shown in $A_{1}$ for all photomicrographs): $100 \mu \mathrm{m}$.

gyrus molecular layer ipsilateral to the lesion (Fig. 4). The two mouse groups that received ES displayed a similar sprouting response after the entorhinal cortex lesion [M-ES from $100 \%$ to $127.9 \pm 8.5 \%(p<0.05)$ and H-ES from $100 \%$ to $118.8 \pm 6.4 \%$ $(p<0.05)$, respectively] (Fig. 5). However, in mice that were
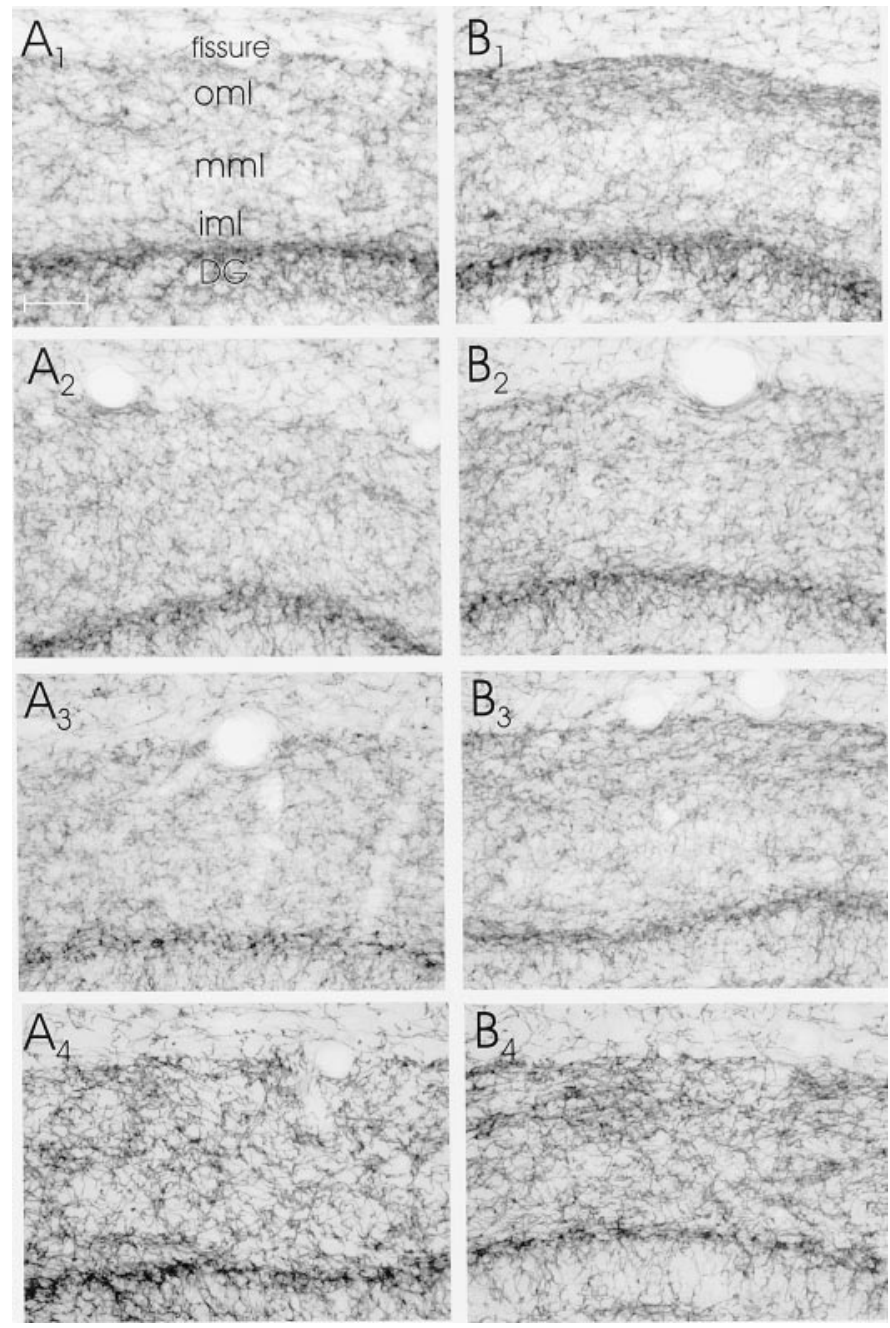

Figure 4. Eight high-power photomicrographs of AchE-stained sections through the dorsal hippocampus. $A$, Contralateral to the entorhinal cortex lesion; $B$, ipsilateral to the entorhinal cortex lesion. $A_{1}, B_{1}$, Sections of an EControl mouse; $A_{2}, B_{2}$, an OE mouse; $A_{3}, B_{3}$, sections from an M-ES mouse; $A_{4}, B_{4}$, sections from an H-ES mouse. $D G$, Dentate gyrus. Scale bar (shown in $A_{1}$ for all photomicrographs): $100 \mu \mathrm{m}$.

ovariectomized and did not receive estrogen supplementation (i.e., the OE group), the sprouting response was substantially reduced (i.e., no change; $100 \%$ and $100.1 \pm 1.2 \%$ ) (Figs. 4, 5). These changes in density, however, were not significantly different between the groups $\left(F_{(3,26)}=2.81 ; p<0.06\right)$, because each group had a few animals in which AChE staining was either not changed or changed very little.

\section{DISCUSSION}

These studies demonstrate that in mice, after an ovariectomy, the axonal sprouting response of the hippocampal formation to lesions of its main cortical input, the entorhinal cortex, is substantially reduced. Estrogen supplementation, both at a moderate dose and at a high dose, restores the sprouting response to the level of the non-ovariectomized, i.e., the normal mouse. In short, lack of estrogen dramatically reduces plasticity (as indicated by the axonal sprouting response) compared with animals with normal (moderate or high) levels of estrogen present in the bloodstream.

After ovariectomy, with its concomitant reduction in estrogen 


\begin{tabular}{|c|c|c|c|c|c|c|}
\hline Mouse group & $\begin{array}{l}\text { DG, ml, } \\
\text { ipsilateral }(\mu \mathrm{m})\end{array}$ & $\begin{array}{l}\text { DG, iml, } \\
\text { ipsilateral }(\mu \mathrm{m})\end{array}$ & $\begin{array}{l}\text { DG, ml, } \\
\text { contralateral }(\mu \mathrm{m})\end{array}$ & $\begin{array}{l}\text { DG, iml, } \\
\text { contralateral }(\mu \mathrm{m})\end{array}$ & $\begin{array}{l}\text { CA1, } \\
\text { ipsilateral }(\mu \mathrm{m})\end{array}$ & $\begin{array}{l}\text { CA1, } \\
\text { contralateral }(\mu \mathrm{m})\end{array}$ \\
\hline ECL & $144 \pm 7$ & $28 \pm 2$ & $148 \pm 6$ & $30 \pm 2$ & $295 \pm 23$ & $284 \pm 21$ \\
\hline $\mathrm{ECL}+\mathrm{OE}$ & $142 \pm 7$ & $25 \pm 1$ & $137 \pm 8$ & $27 \pm 2$ & $277 \pm 18$ & $273 \pm 18$ \\
\hline \multicolumn{7}{|l|}{$\mathrm{ECL}+\mathrm{OE}$} \\
\hline + M-ES & $138 \pm 10$ & $27 \pm 2$ & $142 \pm 6$ & $28 \pm 1$ & $288 \pm 19$ & $283 \pm 13$ \\
\hline \multicolumn{7}{|l|}{$\mathrm{ECL}+\mathrm{OE}$} \\
\hline + H-ES & $153 \pm 5$ & $32 \pm 2$ & $148 \pm 9$ & $31 \pm 2$ & $275 \pm 18$ & $294 \pm 22$ \\
\hline
\end{tabular}

Please note that there is no shrinkage in any layer.

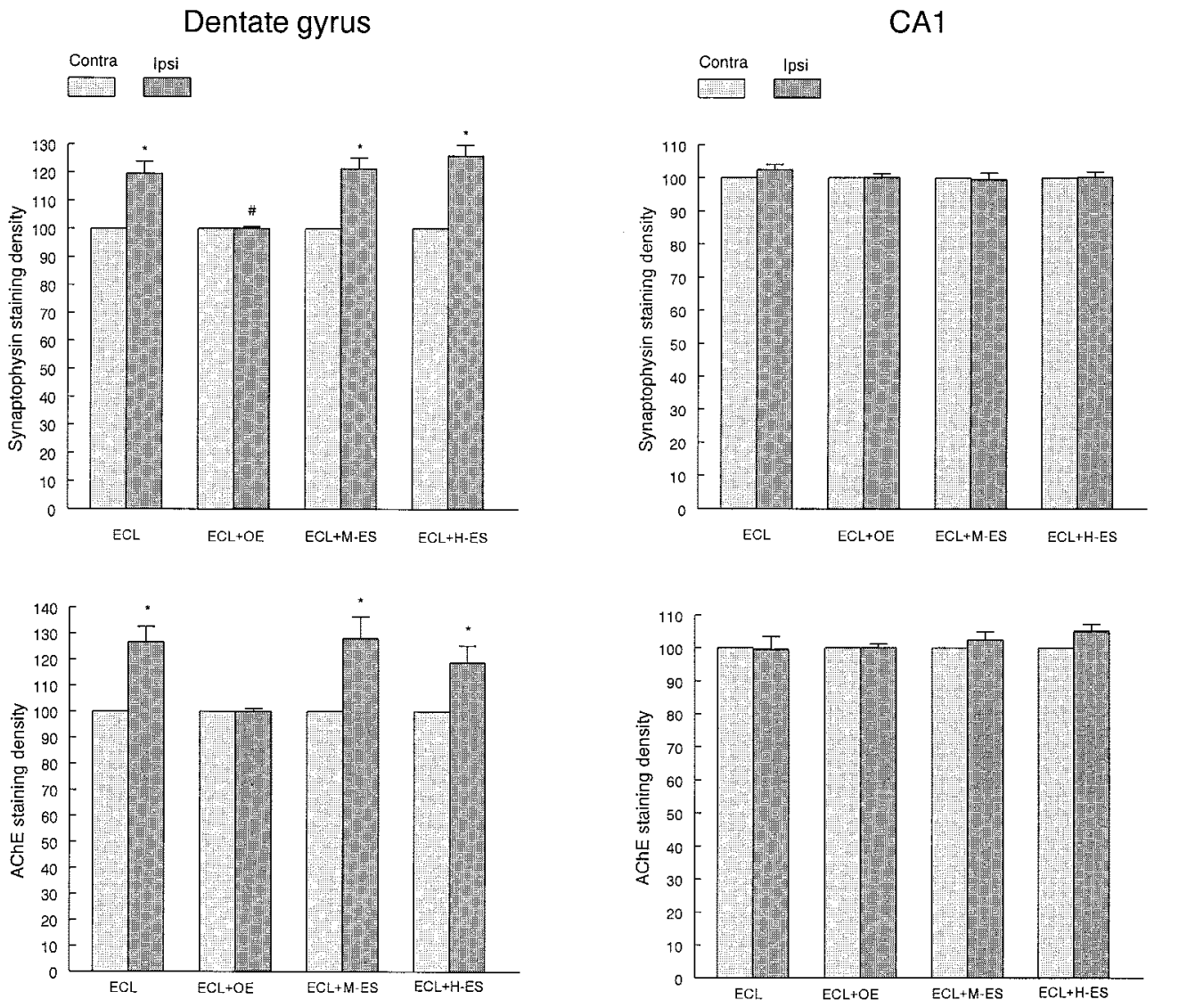

Figure 5. Bar graphs demonstrating the optical density measurements of the synaptophysin and AChE staining in the mouse hippocampus. The contralateral density was set as $100 \%$. ECL, Entorhinal cortex lesion $(n=10) ; O E$, ovariectomy $(n=8) ; M-E S$, moderate estrogen supplementation $(n=8) ; H$-ES, high estrogen supplementation $(n=8)$. ${ }^{*} p<$ 0.01 significantly different from contralateral; \# $p<0.001$ significantly different from OE. levels, the uterine weights of the mice are very much reduced, similar to findings in other studies (Ryan and Schwartz, 1980; Stone et al., 1998). In contrast, the uterine weights are increased after ES compared with normal mice. This is as expected because the moderate dose pellets of estradiol (M-ES) result in blood levels of estrogen slightly above the normal, diestrous levels of estrogen (i.e., 75-100 and $20-40 \mathrm{pg} / \mathrm{ml}$, respectively); the high dose (H-ES) results in estrogen levels somewhat above the normal, proestrous level (i.e., from $\sim 250$ to $300-400 \mathrm{pg} / \mathrm{ml}$, respectively) (Bronson and Desjardins, 1974; Ryan and Schwartz, 1980). Similar to earlier studies, we show that there is a relation between estrogen levels and uterine weight (Bronson and Desjardins, 1974; Ryan and Schwartz, 1980; Stone et al., 1998). We have not measured the blood levels of estrogen in our animals in this study, because in a separate study we have measured these levels (Rissanen et al., 1999), and they have been shown to be similar to the above levels.
The main focus of this study has been to use synaptophysin immunohistochemistry to compare the gross synaptic density in the molecular layer of the dentate gyrus after entorhinal cortex lesions (Calhoun et al., 1996). This technique does not distinguish between synapse types and does not differentiate the identity of the receptor, but it allows us to measure (and compare) presynaptic terminal density over relatively large areas of neuropil. Furthermore, we have only measured changes in the synaptophysin (and AChE) staining density in the dorsal, septal part of the hippocampus, because the axons arising from (the lesioned) lateral parts of the entorhinal cortex terminate predominantly in this part of the hippocampus (Turner et al., 1998; van Groen et al., 2002).

After the lateral entorhinal area lesions, there is an increase in the density of staining for synaptophysin in the outer one-third of the dentate gyrus molecular layer ipsilateral to the lesion; however, because shrinkage of this layer would have affected the 
synaptophysin staining density measurements (Wagner et al., 1983), we measured the width of the dentate gyrus molecular layer. None of the groups of mice showed any change in the width of the dentate gyrus molecular layer or in the width of stratum lacunosum-moleculare + stratum radiatum of area CA1. In contrast, it should be noted that Stone et al. (1998) showed an increase in the width of the outer molecular layer in estrogentreated wild-type mice after perforant path transection.

In earlier experiments, we also established that 4 weeks after the lesion was the earliest time point for measuring unbiased sprouting, i.e., without any interference from degenerative processes (Kadish et al., 2002). The degenerative processes predominantly take place in the first week after the lesion (Matthews et al., 1976a; Gall et al., 1979; Jensen et al., 1994). The degenerating axons and terminals in the molecular layer of the dentate gyrus are removed rapidly by astrocytes and microglia (McWilliams and Lynch, 1979; Steward et al., 1990). Degenerating terminals are being removed within the first day after the lesion by activated microglia (Gall et al., 1979; Jensen et al., 1994). It has been demonstrated that microglia express estrogen receptors (Mor et al., 1999) and that estrogen has anti-inflammatory effects on microglia (Mor et al., 1999; Bruce-Keller et al., 2000). Thus the lack of estrogen could have interfered with the functioning of microglial cells and therefore with the clearance of debris, and this would have hindered regeneration. However, in an earlier study, we have examined the activity of microglia and astrocytes, and there were no differences between normal and OE animals 2 and 4 weeks after entorhinal cortex lesions (Kadish et al., 2002).

The later, "regeneration" phase (which starts $\sim 1$ week after the lesion) is characterized by reinnervation of the denervated zone, i.e., the sprouting of new axons and the formation of new terminals and synapses in the molecular layer of the dentate gyrus (Matthews et al., 1976b; Steward et al., 1990). In our previous studies we have shown that there are no differences in the upregulation of two presynaptically expressed proteins (i.e., GAP43 and synaptophysin) after entorhinal cortex lesions (Kadish et al., 2002). Several electron microscopic studies in the rat have demonstrated that regeneration of synapses is present in the molecular layer of the dentate gyrus after entorhinal cortex lesions (Matthews et al., 1976b; Steward and Vinsant, 1978). Electrophysiological studies have indicated that the growth of new synapses is accompanied by the reestablishment of the entorhinal evoked field potentials in the dentate gyrus (West et al., 1975; Steward et al., 1983). Furthermore, Steward (1982) and Ramirez (1997) have reviewed the functional significance of the entorhinal cortex lesion-induced plasticity in the hippocampus, and they have demonstrated that the time course of the ingrowth of entorhinal cortex axons corresponds with the improvement in behavior and electrophysiological properties.

Several fiber systems have been shown to participate in the reinnervation of the dentate granule cells after entorhinal cortex lesions in rats. Thus, the analysis of the effects of entorhinal cortex lesions on sprouting in the denervated zone of the dentate gyrus is complex, because several fiber systems could sprout into the denervated part of the molecular layer of the dentate gyrus. In the rat, the normally sparse, crossed entorhinal-dentate pathway proliferates within the denervated zone (Zimmer, 1973; Steward et al., 1974; Steward and Vinsant, 1983). However, it should be noted that in the mouse the crossed entorhinal-hippocampal pathway is practically nonexistent (van Groen et al., 2002) and therefore not likely to contribute significantly to the sprouting response. Furthermore, Lynch et al. $(1972,1976)$ and Zimmer
(1973) have shown in young rats that there is an expansion of the terminal field of the CA4 commissural/association system into the zone formerly occupied by the entorhinal axons and terminals after complete entorhinal cortex lesions. West (1984) and Deller and Frotscher (1997) have demonstrated a similar expansion of the commissural fiber system after entorhinal cortex lesions in the adult rat; however, no change in the size of the layer occupied by this system is present in our animals. In contrast, White et al. (2001) have shown an expansion of the inner molecular layer in human apolipoprotein E transgenic mice. It is likely that these differences are (partly) caused by the partial entorhinal cortex lesions (with degeneration of the lateral perforant path only) in this study compared with complete entorhinal cortex lesions in the other studies.

It has been demonstrated that the AchE-containing septohippocampal pathway proliferates within the denervated zone (Lynch et al., 1972; Nadler et al., 1977a,b; Stanfield and Cowan, 1982). Nonetheless, no change in choline acetyltransferase (ChAT) staining is present after the entorhinal cortex lesions in the mouse (Kadish et al., 1999). Furthermore, the change in density of staining for $\mathrm{AChE}$ is not related to an actual increase in axon number (Calhoun and Mouton, 2001; our preliminary studies). High-power analysis of the zone of upregulated AChE staining shows a higher density of staining per axon and clumps of AChE associated with axons; together these indicates a higher expression of AChE per axon. Furthermore, Henderson et al. (1998) have demonstrated in the rat an apparent increase in the density of ChAT-containing terminals after unilateral entorhinal cortex lesion; however, they concluded that this increased density could be accounted for entirely by tissue shrinkage. In contrast, it should be noted that Nyakas et al. (1988) demonstrated an increased innervation from the septum to the hippocampus after unilateral entorhinal cortex lesions in the rat. Taken together, this may indicate that some of the noncholinergic (i.e., GABAergic) septal axons possibly sprout after entorhinal cortex lesions. Ovariectomy per se also influences the cholinergic system. Luine (1985) has shown a significant decrease in ChAT staining in the hippocampus 2 weeks after ovariectomy in the mouse, but Gibbs et al. (1994) have demonstrated that 4 weeks after an ovariectomy the ChAT levels in the hippocampus have returned to normal. We do show, however, a significant increase in the density of staining for AChE after the entorhinal cortex lesion in the mouse, indicating that this enzyme is upregulated in the hippocampus. This would concur with the suggestion that $\mathrm{AChE}$ has a function in regulating plasticity as such (Small, 1989).

The sprouting response in the dentate gyrus after entorhinal cortex lesions is dramatically reduced in ovariectomized animals, suggesting that a lack of estrogen leads to a reduction in plasticity. This hypothesis is corroborated by the results of the ES mouse groups, because the estrogen supplementation in the ovariectomized mice enhanced the regeneration response of the brain to lesions to a level similar to that of normal, non-ovariectomized mice.

Many studies have suggested that estradiol is a trophic hormone in the brain during fetal development (Beyer, 1999), and it has been demonstrated that the adult brain remains highly plastic and hormone regulated (García-Segura et al., 1994; Desmond and Levy, 1997). Estradiol influences this plasticity by acting as an important trophic and protective factor throughout the life span, even in the human brain (Garcia-Estrada et al., 1993; GarcíaSegura et al., 1994; McEwen and Alves, 1999). The neurotrophic effects of estradiol are many: induction of neurite outgrowth, den- 
dritic spines, and synaptogenesis; influence on long term potentiation and excitability; and enhancement of gene expression (García-Segura et al., 1994; Desmond and Levy, 1997; McEwen and Alves, 1999). It has been shown that sex differences are present in the reaction of the rat brain to injury (Loy and Milner, 1980; Morse et al., 1986). Estrogen supplementation has been shown to enhance axonal sprouting and the regeneration of lesioned neuronal processes in the hippocampus of the rat (Morse et al., 1992). Morse et al. (1992) have shown an increase in the width of the commissural/associational pathway after complete entorhinal cortex lesions.

Estradiol may exert its trophic and protective effects by acting via classic genomic mechanisms (i.e., through the estrogen receptor) on various genes, including the neurotrophins and their receptors, cell death proteins and structural proteins (McEwen and Alves, 1999). There are two known estrogen receptors, ER- $\alpha$ and ER- $\beta$, and the colocalization of estrogen receptors, neurotrophins, and their cognate receptors suggests potential interactions between estrogen and neurotrophins (Gibbs et al., 1994). Furthermore, estradiol may exert direct protective effects by modifying blood flow (Mendelsohn, 2000). These effects are both short-term, i.e., direct modulation of vascular smooth muscle cell relaxation, and long-term, i.e., through estrogen receptormediated changes in gene and protein expression (Mendelsohn, 2000). Another pathway through which estrogen could exert its effects is by means of the nongenomic membrane receptors and their signaling pathways (such as cAMP and $\mathrm{IP}_{3}$ ) (Beyer, 1999; McEwen and Alves, 1999).

In summary, our data demonstrate that low levels of estrogen lead to a reduction in the sprouting response in the dentate gyrus after entorhinal cortex lesions; i.e., low estrogen levels lead to a reduction in brain plasticity. Numerous studies from humans and animal models have suggested that estrogen may be beneficial in preserving cognitive function (Desmond and Levy, 1997; Brinton et al., 2000; Henderson et al., 2000). The ability of gonadal steroids to alleviate neurological symptoms has been addressed in human studies (Fillit et al., 1986), and recently it has been reported that estradiol may be beneficial in neurodegenerative diseases such as Alzheimer's disease (Brinton et al., 2000, Henderson et al., 2000). Together, these data may provide a better comprehension of the clinical observations of improved cognitive function and decreased neurodegeneration in women who receive hormone replacement therapy.

\section{REFERENCES}

Beyer C (1999) Estrogen and the developing brain. Anat Embryol 199:379-390.

Brinton RD, Chen S, Montoya M, Hsieh D, Minaya J, Kim J, Chu H-P (2000) The women's health initiative: estrogen replacement therapy is neurotrophic and neuroprotective. Neurobiol Aging 21:475-496.

Bronson FH, Desjardins C (1974) Circulating concentrations of FSH, $\mathrm{LH}$, estradiol, and progesterone associated with acute, male-induced puberty in female mice. Endocrinology 94:1658-1668.

Bruce-Keller AJ, Keeling JL, Keller JN, Huang FF, Camondola S, Mattson MP (2000) Anti-inflammatory effects of estrogen on microglial activation. Endocrinology 141:3646-3656.

Calhoun ME, Mouton PR (2001) Length measurement: new developments in neurostereology and $3 \mathrm{D}$ imagery. $\mathrm{J}$ Chem Neuroanat 20:61-69.

Calhoun ME, Jucker M, Martin LJ, Thinakaran G, Price DL, Mouton PR (1996) Comparative evaluation of synaptophysin-based methods for quantification of synapses. J Neurocytol 25:821-828.

Cotman CW, Nadler JV (1978) Reactive synaptogenesis in the hippocampus. In: Neuronal plasticity (Cotman CW, ed), pp 227-271. New York: Raven.

Deller T, Frotscher M (1997) Lesion-induced plasticity of central neurons: sprouting of single fibers in the rat hippocampus after unilateral entorhinal cortex lesions. Prog Neurobiol 53:687-727.
Desmond NL, Levy WB (1997) Ovarian steroidal control of connectivity in the female hippocampus: an overview of recent experimental findings and speculations on its functional consequences. Hippocampus 7:239-245.

Dubal DB, Wilson ME, Wise PM (1999) Estradiol: a protective and trophic factor in the brain. Alzheimer's Dis Rev 4:1-9.

Fillit H, Weinreb H, Cholst I, Luine V, McEwen B, Amador R, Zabriskie $\mathrm{J}$ (1986) Observations in a preliminary open trial of estradiol therapy for senile dementia-Alzheimer's type. Psychoneuroendocrinology 11:337-345.

Frotscher M, Heimrich B, Deller T (1997) Sprouting in the hippocampus is layer-specific. Trends Neurosci 20:218-223.

Gall C, Rose G, Lynch G (1979) Proliferative and migratory activity of glial cells in the partially deafferented hippocampus. J Comp Neurol 183:539-550.

Garcia-Estrada J, Del Rio JA, Luquin S, Soriano E, García-Segura LM (1993) Gonadal hormones down-regulate reactive gliosis and astrocyte proliferation after penetrating brain injury. Brain Res 628271-278.

García-Segura LM, Chowen JA, Párducz A, Naftolin F (1994) Gonadal hormones as promotors of structural synaptic plasticity: cellular mechanisms. Prog Neurobiol 44:279-307.

García-Segura LM, Azcoitia I, DonCarlos LL (2001) Neuroprotection by estradiol. Prog Neurobiol 63:29-60.

Gibbs RB, Wu D, Hersh LB, Pfaff DW (1994) Effects of estrogen replacement on the relative levels of choline acetyltransferase, trkA, and the nerve growth factor messenger RNAs in the basal forebrain and hippocampal formation of adult rats. Exp Neurol 129:70-80.

Henderson VW, Paganini-Hill A, Miller BL, Elble RJ, Reyes PF, Shoupe D, McCleary CA, Klein AR, Hake AM, Farlow MR (2000) Estrogen for Alzheimer's disease in women: randomized, double blind, placebo controlled trial. Neurology 54:295-301.

Henderson Z, Harrison PS, Jagger E, Beeby JH (1998) Density of choline acetyltransferase-immunoreactive terminals in the rat dentate gyrus after entorhinal cortex lesions: a quantitative light microscopic study. Exp Neurol 152:50-63.

Hyman BT, Van Hoesen GW, Kromer LJ, Damasio AR (1986) Perforant pathway changes and the memory impairment of Alzheimer's disease. Ann Neurol 20:472-481.

Hyman BT, Kromer LJ, Van Hoesen GW (1988) A direct demonstration of the perforant pathway terminal zone in Alzheimer's disease using the monoclonal antibody Alz-50. Brain Res 450:392-397.

Jensen MB, Gonzalez B, Castellano B, Zimmer J (1994) Microglial and astroglial reactions to anterograde axonal degeneration: a histochemical and immunocytochemical study of the adult rat fascia dentata after entorhinal perforant path lesions. Exp Brain Res 98:245-260.

Kadish I, van Groen T, Riekkinen Jr P (1999) Differences in entorhinal cortex lesion-induced sprouting in the hippocampus between rats and mice. Soc Neurosci Abstr 25:2030.

Kadish I, Pradier L, van Groen T (2002) Transgenic mice expressing the human presenilin 1 gene demonstrate enhanced hippocampal reorganization following entorhinal cortex lesions. Brain Res Bull 57:587-594.

Loy R, Milner TA (1980) Sexual dimorphism in extent of axonal sprouting in rat hippocampus. Science 208:1282-1284.

Luine VN (1985) Estradiol increases choline acetyltransferase activity in specific basal forebrain nuclei and projection areas of female rats. Exp Neurol 89:484-490.

Lynch G, Matthews DA, Mosko S, Parks T, Cotman C (1972) Induced acetylcholinesterase-rich layer in rat dentate gyrus following entorhinal lesions. Brain Res 42:311-318.

Lynch G, Gall C, Rose G, Cotman CW (1976) Changes in the distribution of the dentate gyrus associational system following unilateral or bilateral entorhinal lesion in the adult rat. Brain Res 110:57-71.

Matthews DA, Cotman C, Lynch G (1976a) An electron microscopic study of lesion-induced synaptogenesis in the dentate gyrus of the adult rat. I. Magnitude and time course of degeneration. Brain Res 115:1-21.

Matthews DA, Cotman C, Lynch G (1976b) An electron microscopic study of lesion-induced synaptogenesis in the dentate gyrus of the adult rat. II. Reappearance of morphologically normal synaptic contacts. Brain Res 115:23-41.

McEwen BS, Alves SE (1999) Estrogen actions in the central nervous system. Endocr Rev 20:279-307.

McWilliams R, Lynch G (1979) Terminal proliferation in the partially deafferented dentate gyrus: time courses for the appearance and removal of degeneration and the replacement of lost terminals. J Comp Neurol 187:191-198.

Mendelsohn ME (2000) Mechanisms of estrogen action in the cardiovascular system. J Steroid Biochem Mol Biol 74:337-343.

Mor G, Nilsen J, Horvath T, Bechmann I, Brown S, García-Segura LM, Naftolin F (1999) Estrogen and microglia: a regulatory system that affects the brain. J Neurobiol 40:484-496.

Morse JK, Scheff SW, DeKosky ST (1986) Gender steroids influence axon sprouting in the hippocampal dentate gyrus: a sexually dimorphic response. Exp Neurol 94:649-658.

Morse JK, Scheff SW, DeKosky ST (1992) Neurotrophic effects of ste- 
roids on lesion-induced growth in the hippocampus. II. Hormone replacement. Exp Neurol 118:47-52.

Nadler JV, Cotman CW, Lynch GS (1977a) Histochemical evidence of altered development of cholinergic fibers in the rat dentate gyrus following lesions. I. Time course after complete unilateral entorhinal lesion at various ages. J Comp Neurol 171:561-588.

Nadler JV, Cotman CW, Lynch GS (1977b) Histochemical evidence of altered development of cholinergic fibers in the rat dentate gyrus following lesions. II. Effects of partial entorhinal and simultaneous multiple lesions. J Comp Neurol 171:589-604.

Nyakas C, Luiten PGM, Balkan B, Spencer Jr DG (1988) Changes in septo-hippocampal projections after lateral entorhinal or combined entorhinal-raphé lesions as studied by anterograde tracing methods. Brain Res Bull 21:285-293.

Ramirez JJ (1997) The functional significance of lesion-induced plasticity of the hippocampal formation. Adv Neurol 73:61-82.

Rissanen A, Puoliväli J, van Groen T, Riekkinen Jr P (1999) In mice tonic estrogen replacement therapy improves non-spatial and spatial memory in a water maze task. NeuroReport 10:1369-1372.

Ryan KD, Schwartz NB (1980) Changes in the serum hormone levels associated with male-induced ovulation in group-housed adult female mice. Endocrinology 106:959-966.

Shugrue P, Scrimo P, Lane M, Askew R, Merchentaler I (1997) The distribution of estrogen receptor- $\beta$ mRNA in forebrain regions of the estrogen- $\alpha$ knockout mouse. Endocrinology 138:5649-5652.

Small DH (1989) Acetylcholinesterases: zymogens of neuropeptide processing enzymes? Neuroscience 29:241-249.

Stanfield BB, Cowan WM (1982) The sprouting of the septal afferents to the dentate gyrus after lesions of the entorhinal cortex in adult rats. Brain Res 232:162-170.

Steward O (1982) Assessing the functional significance of lesion-induced neuronal plasticity. Int Rev Neurobiol 23:197-254.

Steward O (1991) Synapse replacement on cortical neurons following denervation. In: Cerebral cortex, Vol 9 (Peters A, Jones EG, eds), pp 81-132. New York: Kluwer Academic/Plenum.

Steward O, Vinsant SL (1983) The process of reinnervation in the dentate gyrus of the adult rat: a quantitative electron microscopic analysis of terminal proliferation and reactive synaptogenesis. J Comp Neurol 214:370-386.

Steward O, Vinsant SL (1987) Collateral projections of cells in the surviving entorhinal area which reinnervate the dentate gyrus of the rat following entorhinal lesions. Brain Res 149:216-222.

Steward O, Cotman CW, Lynch GS (1973) Re-establishment of electrophysiologically functional entorhinal cortical input to the dentate gyrus deafferented by ipsilateral entorhinal lesion: innervation by the contralateral entorhinal cortex. Exp Brain Res 18:396-414.

Steward O, Cotman CW, Lynch GS (1974) Growth of a new fiber projection in the brain of adult rats: re-innervation of the dentate gyrus by the contralateral entorhinal cortex following ipsilateral entorhinal lesions. Exp Brain Res 20:45-66.

Steward O, Torre ER, Phillips LL, Trimmer PA (1990) The process of reinnervation in the dentate gyrus of adult rats: time course of increases in mRNA for glial fibrillary acidic protein. J Neurosci 10:2373-2384.

Stone DJ, Rozovsky I, Morgan TE, Anderson CP, Finch CE (1998) Increased synaptic sprouting in response to estrogen via an apolipoprotein E-dependent mechanism: implications for Alzheimer's disease. J Neurosci 19:3180-3185.

Turner DA, Buhl EH, Hailer NP, Nitsch R (1998) Morphological features of the entorhinal-hippocampal connection. Prog Neurobiol 55:537-562.

van Groen T, Wyss JM (1992) Projections from the laterodorsal nucleus of the thalamus to the limbic and visual cortices in the rat. J Comp Neurol 324:427-448.

van Groen T, Miettinen P, Kadish I (2002) The entorhinal cortex of the mouse. Topographic organization of the projections to the hippocampus. Hippocampus, in press.

Wagner GP, Oertel WH, Wolff JR (1983) Entorhinal lesions result in shrinkage of the outer molecular layer of rat dentate gyrus leading subsequently to an apparent increase of glutamate decarboxylase and cytochrome oxidase activities. Neurosci Lett 39:255-260.

West JR (1984) Age-dependent sprouting in the dentate gyrus demonstrated with anterograde HRP. Brain Res Bull 12:323-330.

West JR, Deadwyler S, Cotman CW, Lynch G (1975) Time-dependent changes in commissural field potentials in the dentate gyrus following lesions of the entorhinal cortex in adult rats. Brain Res 97:215-233.

White F, Nicoll JA, Horsburgh K (2001) Alterations in ApoE and ApoJ in relation to degeneration and regeneration in a mouse model of entorhinal cortex lesion. Exp Neurol 169:307-318.

Zimmer J (1973) Extended commissural and ipsilateral projections in postnatally de-entorhinated hippocampus and fascia dentata demonstrated in rats by silver impregnation. Brain Res 64:293-311. 